\title{
Magnetic Fields in the Milky Way Halo
}

\author{
S. A. $\mathrm{Mao}^{1,2}$, N. M. McClure-Griffiths ${ }^{3}$, B. M. Gaensler ${ }^{4}$, \\ J. C. Brown ${ }^{5}$, C. L. van Eck ${ }^{5}$, M. Haverkorn ${ }^{6}$, P. P. Kronberg ${ }^{7,8}$, \\ J. M. Stil ${ }^{5}$, A. Shukurov ${ }^{9}$ and A. R. Taylor ${ }^{5}$ \\ ${ }^{1}$ Jansky Fellow, National Radio Astronomy Observatory, \\ P.O. Box O, Socorro, NM 87801, USA \\ email: mao@astro.wisc.edu \\ ${ }^{2}$ Department of Astronomy, University of Wisconsin, Madison, WI 53706, USA \\ ${ }^{3}$ CSIRO Astronomy and Space Science, Australia Telescope National Facility, \\ PO Box 76, Epping, NSW 1710, Australia \\ ${ }^{4}$ Sydney Institute for Astronomy, School of Physics, \\ The University of Sydney, NSW 2006, Australia \\ ${ }^{5}$ Department of Physics and Astronomy, and Institute for Space Imaging Science, \\ University of Calgary, AB, Canada \\ ${ }^{6}$ Department of Astrophysics, Radboud University, \\ P.O. Box 9010, 6500 GL Nijmegen, The Netherlands \\ ${ }^{7}$ Los Alamos National Laboratory, P.O. Box 1663, Los Alamos, NM 87545, USA \\ ${ }^{8}$ Department of Physics, University of Toronto, 60 St. George Street, \\ Toronto, M5S 1A7, Canada \\ ${ }^{9}$ School of Mathematics and Statistics, University of Newcastle, \\ Newcastle upon Tyne, NE1 7RU, UK
}

\begin{abstract}
We present a study of the Milky Way halo magnetic field, determined from observations of Faraday rotation measure (RM) of extragalactic radio sources (EGS) in Galactic longitude range $100^{\circ}-117^{\circ}$ within $30^{\circ}$ of the Galactic plane. We find negative median RMs in both the northern and southern Galactic hemispheres for $|b|>15^{\circ}$, outside the latitude range where the disk field dominates. This suggest that the halo magnetic field towards the outer Galaxy does not reverse direction across the mid-plane. An azimuthal magnetic field at heights $0.8-2 \mathrm{kpc}$ above/below the Galactic plane between the local and the Perseus spiral arm can reproduce the observed trend of RM against Galactic latitude. We propose that the Milky Way could have a halo magnetic field similar to that observed in M51.
\end{abstract}

Keywords. Galaxy: halo, ISM: magnetic fields, polarization

We describe a study of the Galactic halo magnetic field towards the Perseus arm in the second Galactic quadrant using Faraday rotation measure of extragalactic radio sources. This work is based on 302 new EGS RMs measured using the Very Large Array and 339 EGSs RMs from the Canadian Galactic Plane Survey latitude extension region (J. C. Brown et al. in preparation). For $|b|<15^{\circ}$, we find a symmetric RM distribution about the mid-plane, which is consistent with an even parity disk magnetic field. For $|b|>15^{\circ}$, we demonstrate that none of the existing halo magnetic field models can reproduce the observed RM distribution. We then propose a simple halo magnetic field model that can reproduce the behavior of RM as a function of Galactic latitude in the observed region. We suggest that the Milky Way halo magnetic field could have a geometry similar to that observed in M51 (Mao et al. 2012).

\section{References}

Mao, S. A., McClure-Griffiths, N. M., Gaensler, B. M., Brown, J. C., van Eck, C. L., Haverkorn, M., Kronberg, P. P., Stil, J. M., Shukurov, A., \& Taylor, A. R. 2012, ApJ 755, 21 